九州大学学術情報リポジトリ

Kyushu University Institutional Repository

Positive effect of exogenous brain-derived neurotrophic factor on impaired neurite development and mitochondrial function in dopaminergic neurons derived from dental pulp stem cells from children with attention deficit hyperactivity disorder

フン, ティ, ヌイン, ヌイン

https://doi.org/10.15017/2556296

出版情報：九州大学，2019，博士（歯学），課程博士 バージョン：

権利関係 : 


\title{
Positive effect of exogenous brain-derived neurotrophic factor on impaired neurite development and mitochondrial function in dopaminergic neurons derived from dental pulp stem cells from children with attention deficit hyperactivity disorder
}

\author{
Huong Thi Nguyen Nguyen ${ }^{a, 1}$, Hiroki Kato ${ }^{a,{ }^{* *}, 1}$, Hiroshi Sato ${ }^{a}$, Haruyoshi Yamaza ${ }^{a}$, \\ Yasunari Sakai ${ }^{\text {, }}$, Shouichi Ohga ${ }^{\text {, }}$, Kazuaki Nonaka ${ }^{a}$, Keiji Masuda ${ }^{\text {a, }}$ \\ a Section of Oral Medicine for Children, Division of Oral Health, Growth \& Development, Faculty of Dental Science, Kyushu University, Maidashi 3-1-1, \\ Higashi-Ku, Fukuoka, 812-8582, Japan \\ b Department of Pediatrics, Graduate School of Medical Sciences, Kyushu University, Maidashi 3-1-1, Higashi-Ku, Fukuoka, 812-8582, Japan
}

\section{A R T I C L E I N F O}

\section{Article history:}

Received 31 March 2019

Accepted 11 April 2019

Available online 19 April 2019

\section{Keywords:}

Attention deficit hyperactivity disorder

Dopaminergic neurons

Mitochondria

Brain-derived neurotrophic factor

Human exfoliated deciduous teeth stem cells

\begin{abstract}
A B S T R A C T
Attention deficit hyperactivity disorder (ADHD) is one of the most common neurodevelopmental disorders and is characterized by impaired attention, hyperactivity, and impulsivity. While multiple etiologies are implicated in ADHD, its underlying mechanism(s) remain unclear. Although previous studies have suggested dysregulation of dopaminergic signals, mitochondria, and brain-derived neurotrophic factor (BDNF) in ADHD, few studies have reported these associations directly. Stem cells from human exfoliated deciduous teeth (SHED) can efficiently differentiate into dopaminergic neurons (DNs) and are thus a useful disease-specific cellular model for the study of neurodevelopmental disorders associated with DN dysfunction. This study aimed to elucidate the relationships between DNs, mitochondria, and BDNF in ADHD by analyzing DNs differentiated from SHED obtained from three boys with ADHD and comparing them to those from three typically developing boys. In the absence of exogenous BDNF in the cell culture media, DNs derived from boys with ADHD (ADHD-DNs) exhibited impaired neurite outgrowth and branching, decreased mitochondrial mass in neurites, and abnormal intracellular ATP levels. In addition, BDNF mRNA was significantly decreased in ADHD-DNs. Supplementation with BDNF, however, significantly improved neurite development and mitochondrial function in ADHD-DNs. These results suggest that ADHD-DNs may have impaired neurite development and mitochondrial function associated with insufficient production of BDNF, which may be improved by exogenous BDNF supplementation. Findings such as these, from patient-derived SHED, may contribute to the future development of treatment strategies for aberrant dopaminergic signaling, mitochondrial functioning, and BDNF levels implicated in ADHD pathogenesis.
\end{abstract}

() 2019 Elsevier Inc. All rights reserved.

\section{Introduction}

Attention deficit hyperactivity disorder (ADHD) is characterized

Abbreviations: ADHD, Attention deficit hyperactivity disorder; BDNF, brainderived neurotrophic factor; DN, Dopaminergic neuron; SHED, Stem cells from human exfoliated deciduous teeth.

* Corresponding author.

** Corresponding author.

E-mail addresses: kato@dent.kyushu-u.ac.jp (H. Kato), kemasuda@dent.kyushuu.ac.jp (K. Masuda).

1 These authors contributed equally to this study. by inappropriate inattention, hyperactivity, and impulsivity, and is one of the most common neurodevelopmental disorders [1]. ADHD is associated with impaired development of neuronal networks in the brain, which persist from early developmental into adulthood [2,3]. The etiology of ADHD and its risk factors may involve genetic factors, environmental factors, or their interaction [4,5]. However, the neurobiological mechanism(s) underlying ADHD remain unclear, in part because of the extreme heterogeneity of ADHD symptoms, their severity, and the clinical courses which ADHD can assume. The limitations of existing in vivo approaches to the study of living human brains and animal models have also posed a challenge to advancing ADHD treatment [1]. 
Dopaminergic signals play important roles in motor control, cognitive function, and attention, all of which are closely related to the core symptoms of ADHD [6,7]. Previous studies have reported altered dopamine metabolism and dopamine receptor expression profiles in ADHD brain tissues [4,7,8]. Furthermore, therapeutic agents that increase dopamine utilization in the brain are often effective for the treatment of ADHD [9]. Another study reported mitochondrial dysfunction in patients with ADHD [10]. In addition, ADHD is associated with altered brain-derived neurotrophic factor (BDNF) functioning, which plays important roles in neuronal growth, survival, and plasticity as an autocrine or paracrine factor [11-14]. Thus, dopaminergic signals, mitochondrial function, and BDNF signaling are strongly implicated in the pathogenesis of ADHD. However, few studies have demonstrated the neurobiological relationships between these factors.

Stem cells from human exfoliated deciduous teeth (SHED) are neural crest-derived mesenchymal stem cells and efficiently differentiate into dopaminergic neurons (DNs) [15,16]. To better understand the pathogenesis of various neurodevelopmental disorders, our group has previously analyzed SHED obtained from children with these disorders [17-19]. In particular, SHED are a useful disease-specific cellular model for the study of DN-related neuropathology in ADHD. The purpose of the present study was to clarify the pathological association between DNs, mitochondria, and BDNF in ADHD. To this effect, SHED were obtained from three boys with ADHD and three typically developing boys. The neurobiological features of DNs differentiated from these SHED were analyzed and compared between the groups.

\section{Materials and methods}

\subsection{Isolation and SHED culture}

Experiments using human samples were reviewed and approved by the Kyushu University Institutional Review Board for Human Genome/Gene Research (permission number: 678-00) and were conducted in accordance with the Declaration of Helsinki. Informed consent was obtained from the patients' guardians prior to study commencement, as all participating patients were minor children.

Deciduous teeth were collected from three typically developing boys (two six-year-olds and one seven-year-old) and three children with ADHD (six, eight, and nine years old boy, respectively). None of these three ADHD children had associated comorbid conditions such as autism spectrum disorder, chromosomal defects, epilepsy, or other Mendelian disorders. SHED were isolated as has previously been described [19] and were cultured in alpha modification of Eagle's Medium (Nacalai Tesque, Kyoto, Japan) with 15\% fetal bovine serum (Sigma-Aldrich, MO, USA), $100 \mu \mathrm{M}$ L-ascorbic acid 2phosphate (Wako Pure Chemical Industries, Osaka, Japan), $250 \mu \mathrm{g} /$ $\mathrm{mL}$ fungizone (Life Technologies, NY, USA), $100 \mathrm{U} / \mathrm{mL}$ penicillin, and $100 \mu \mathrm{g} / \mathrm{mL}$ streptomycin (Nacalai Tesque) at $37^{\circ} \mathrm{C}$ in an atmosphere containing $5 \% \mathrm{CO}_{2}$. Cells were used for further experiments as a heterogeneous cell population, according to experimental procedures reported by previous studies [17-19].

\subsection{Differentiation of SHED into DN}

SHED differentiation into DNs was induced as described previously [16,19]. Briefly, $3 \times 10^{5}$ SHED were plated onto a six-well culture plate containing the culture medium described above and incubated overnight at $37{ }^{\circ} \mathrm{C}$ in an atmosphere containing $5 \% \mathrm{CO}_{2}$. Cells were then cultured in serum-free Dulbecco's Modified Eagle's Medium (Nacalai Tesque) supplemented with $20 \mathrm{ng} / \mathrm{mL}$ epidermal growth factor (Sigma-Aldrich), $20 \mathrm{ng} / \mathrm{mL}$ basic fibroblast growth factor (Peprotech, NJ, USA), and 1\% N2 supplement (Life Technologies) for two days at $37^{\circ} \mathrm{C}$ in an atmosphere containing $5 \% \mathrm{CO}_{2}$. In the second step, DMEM was replaced with neurobasal media (Life Technologies) supplemented with 2\% B27 supplement (Life Technologies), $1 \mathrm{mM}$ dibutyryladenosine 3,5-cyclic monophosphate (Nacalai Tesque), $0.5 \mathrm{mM}$ 3-isobutyl-1-methylxanthine (SigmaAldrich), and $200 \mu \mathrm{M}$ ascorbic acid (Nacalai Tesque). Cells were then incubated for five days at $37^{\circ} \mathrm{C}$ in an atmosphere containing $5 \% \mathrm{CO}_{2}$. Additionally, the differentiation of SHED was performed in the presence or absence of $50 \mathrm{ng} / \mathrm{mL}$ BDNF (Peprotech) in the second step.

\subsection{Immunocytochemistry}

DNs were cultured on cover glass and fixed with $4 \%$ paraformaldehyde in $0.1 \mathrm{M}$ phosphate buffered saline (PBS, $\mathrm{pH} 7.4$ ) for 10 min then subsequently permeabilized with $0.1 \%$ Triton X-100 for 5 min. Cells were blocked with $2 \%$ bovine serum albumin in PBS for 20 min then incubated with one of the following primary antibodies for 90 min: anti-Tom20 (Santa Cruz Biotechnology, CA, USA) or anti-tyrosine hydroxylase (TH; Proteintech, IL, USA). Cells were subsequently incubated with Alexa Fluor-conjugated secondary antibodies (Life Technologies). After staining with secondary antibodies in the dark for $60 \mathrm{~min}$, nuclei were counterstained with DAPI (Dojindo, Kumamoto, Japan). Cover glass was then mounted on slides using ProLong Diamond mounting medium (Life Technologies).

\subsection{Analyses of neurite outgrowth and branching in cultured DNs}

Fluorescence images of DNs stained with TH and DAPI were acquired using a Nikon C2 confocal microscope (Nikon, Tokyo, Japan), followed by measurement of the maximum length and total number of neurite branches of each double-positive cell. This measurement was performed using the Neurite Outgrowth module in the MetaMorph software (Molecular Devices, CA, USA).

\subsection{Measurement of mitochondria-stained area per cell area}

Fluorescence images of DN stained with Tom20, TH, and DAPI were acquired using a Nikon C2 confocal microscope (Nikon). The Tom20-stained and TH-stained areas of each DN were then measured as the mitochondrial and cell areas, respectively. Areas were measured using the Multi Wavelengths Cell Scoring module in the MetaMorph software (Molecular Devices).

\subsection{Analysis of intracellular ATP levels}

Cells were harvested in ice-cold PBS. The CellTiter-Glo Luminescent Cell Viability Assay (Promega, WI, USA) was then used to measure intracellular ATP levels.

\subsection{Quantitative reverse transcription polymerase chain reaction $(R T-q P C R)$}

Total RNA extraction and RT-qPCR were performed as previously described [19]. The sequences of the primer used in this study were as follows: NURR1, 5'-GCACTTCGGCAGAGTTGAATGA-3' (forward) and 5'-GGTGGCTGTGTTGCTGGTAGTT-3' (reverse); BDNF, 5'-GGGAA AAGGGAACAGGAAAA- $3^{\prime}$ (forward) and 5'-AACAGACAGGATGGGCA GAA-3' (reverse); and 18S rRNA, 5'-CGGCTACCACATCCAAGGAA-3' (forward) and 5'-GCTGGAATTACCGCGGCT-3' (reverse). The relative expression of the target gene was analyzed using the comparative threshold cycle method by normalizing to $18 \mathrm{~S}$ rRNA expression. 


\subsection{Statistical analyses}

Statistical analyses were performed using Student's $t$-tests. Values are presented as means \pm standard error of the mean (SEM). $P<0.05$ was considered to indicate statistical significance.

\section{Results}

\subsection{Impaired neurite development in DNs differentiated from SHEDs of children with ADHD}

SHED from three typically developing children and three children with ADHD were induced to differentiate into DNs (Ctrl-DNs and ADHD-DNs, respectively) in media conditioned without BDNF (Fig. 1A). There were no significant differences in the expression of NURR1, a marker of DNs in the central nervous system, or in the number of cells expressing $\mathrm{TH}$, a differentiated DN marker, between ADHD-DNs and Ctrl-DNs (Fig. 1B and C). However, when compared with Ctrl-DNs, the maximum neurite length was significantly shorter and the number of neurite branches was significantly reduced in ADHD-DNs (Fig. 1D and E). These results suggested that, without exogenous BDNF, ADHD-DNs had impaired neurite development after differentiation.

\subsection{Impaired mitochondrial activity in DNs differentiated from SHEDs of children with $A D H D$}

Mitochondrial activity and localization in neurites play important roles in neurite development. We examined whether impaired neurite development in ADHD-DNs was associated with mitochondrial dysfunction under conditions without BDNF supplementation. The number of neurites including mitochondria was significantly lower in ADHD-DNs than in Ctrl-DNs (Fig. 2A and B and Suppl. Fig. 1). The mitochondrial area per cell area was also significantly reduced in ADHD-DNs compared with Ctrl-DNs (Fig. 2A, C and Suppl. Fig. 1). Intracellular ATP levels in ADHDDNs were significantly lower than those in Ctrl-DNs (Fig. 2D). These results suggested that the impaired neurite outgrowth seen in ADHD-DNs without exogenous BDNF might be associated with reduced mitochondrial mass, motility, and ATP production.

\subsection{Reduced BDNF mRNA expression and positive effects of exogenous BDNF on neurite development in ADHD-DNs}

Autocrine or paracrine action of endogenous BDNF in neurons is essential for neurite development. To determine whether there were defects in this action in ADHD-DNs, the expression of BDNF was examined. The expression of BDNF in ADHD-DNs was significantly reduced compared with that in Ctrl-DNs (Fig. 3A). In addition, no significant bias was found for the Val66Met polymorphism, which might affect the intracellular transport and secretion of BDNF in these subjects (Suppl. Fig. 2). In cell media supplemented with BDNF, however, neurite outgrowth and branching were significantly enhanced in ADHD-DNs (Fig. 3B-E). These results suggested that impaired neurite development in ADHD-DNs might be associated with reduced endogenous BDNF action predominantly due to transcriptional dysregulation of BDNF, which may be ameliorated by exogenous BDNF.

\subsection{Positive effects of exogenous BDNF on mitochondrial activity in ADHD-DNs}

Next, we examined whether the effects of exogenous BDNF on neurite development in ADHD-DNs involved mitochondrial
A

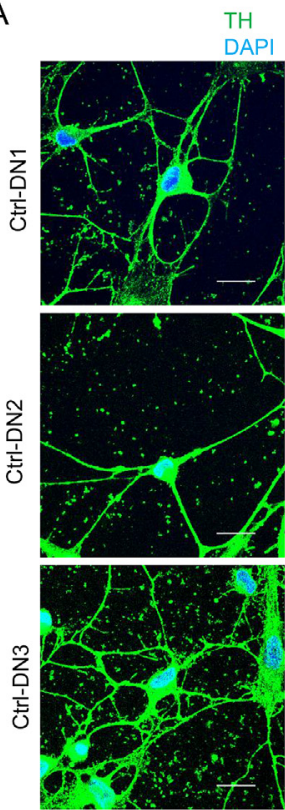

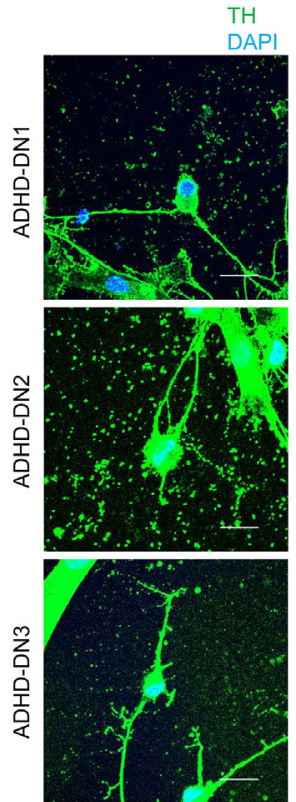

B

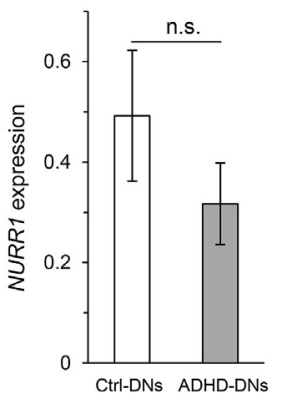

D

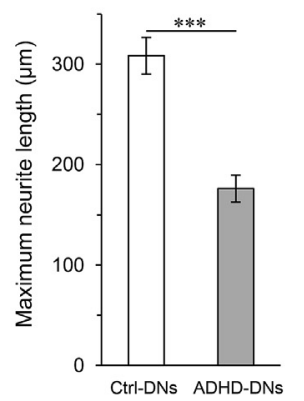

C

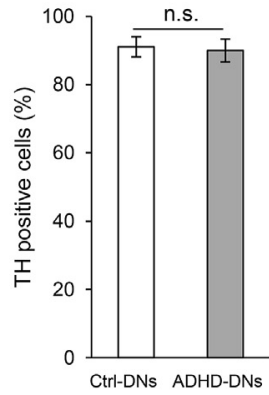

E

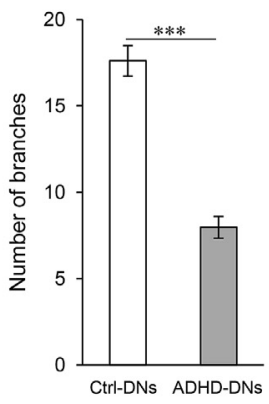

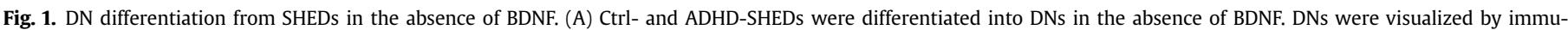

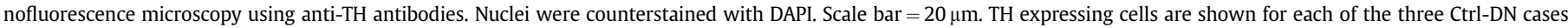

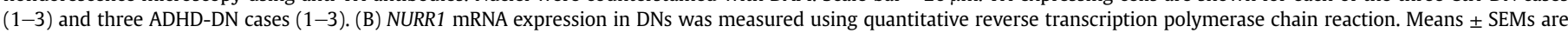

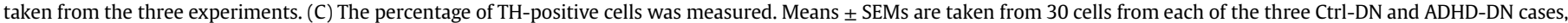

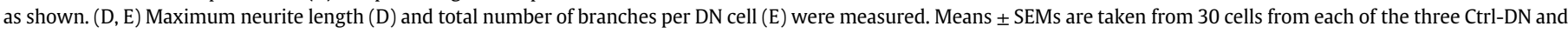
ADHD-DN cases, as shown. n.s., not significant. ${ }^{* * *} P<0.001$. 

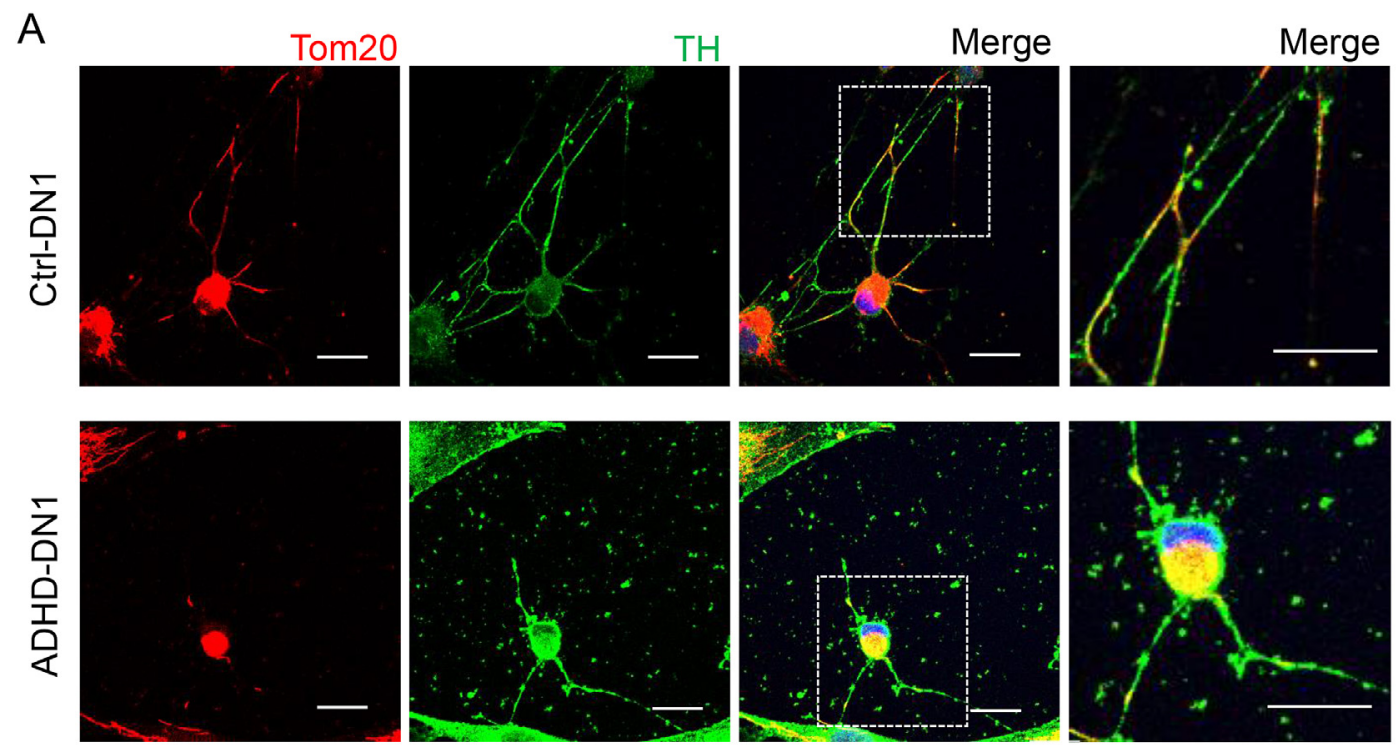

B

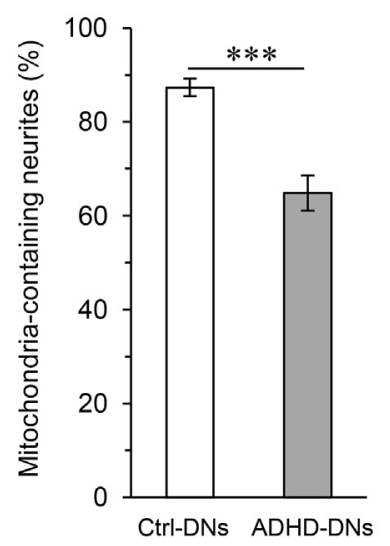

C

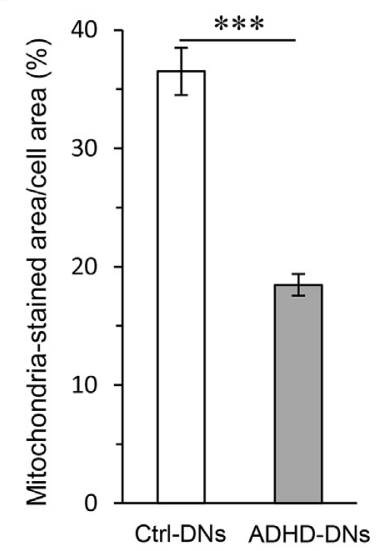

D

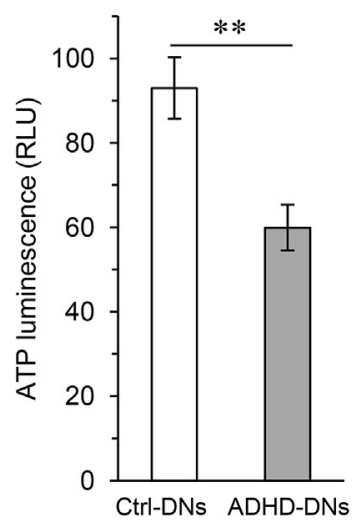

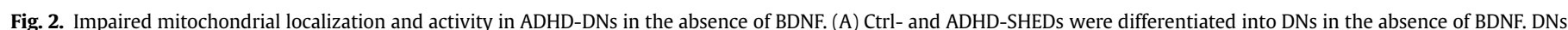

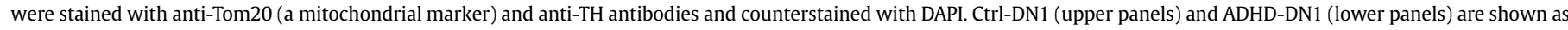

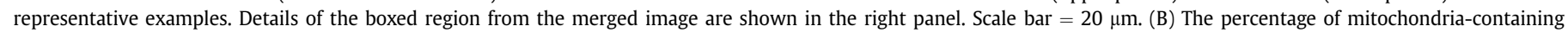

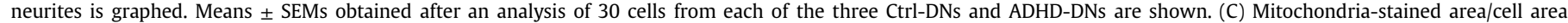

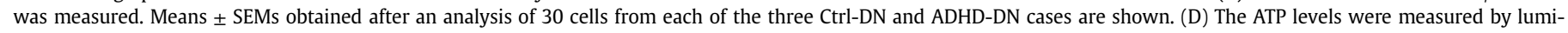

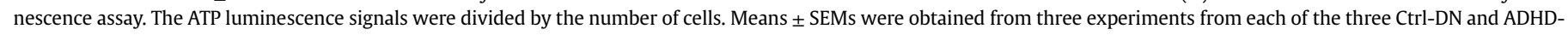
DN cases. ${ }^{* *} P<0.01$. ${ }^{* * *} P<0.001$.

activation. The addition of BDNF to culture media significantly increased the mitochondrial area per cell area, the number of mitochondria-containing neurites, and intracellular ATP levels in ADHD-DNs (Fig. 4A-E, and Suppl. Fig. 3). These results suggest that exogenous BDNF has positive effects on ADHD-DN and improves mitochondrial function, as is required for proper neurite development.

\section{Discussion}

This study aimed to elucidate the association between DNs, mitochondria, and BDNF in the pathogenesis of ADHD. To accomplish this, ADHD-DNs were examined neurobiologically and compared with Ctrl-DNs. ADHD-DNs exhibited impaired neurite development, which was associated with mitochondrial dysfunction (e.g., reduced mitochondrial mass, abnormal localization within neurites, and altered ATP production). Furthermore, we found reduced $B D N F$ expression in ADHD-DNs, which may result in impaired secretion of endogenous BDNF. Finally, we observed that the cellular phenotypes observed in ADHD-DNs were improved by exogenous BDNF administration.

In addition to the media used for differentiating SHED into mature DNs in vitro by Fujii et al. [16], media lacking BDNF was also used in the present study [17,19]. BDNF supplementation of the culture media promotes the maturation of DNs after differentiation [16]. On the other hand, BDNF has been demonstrated to act cellautonomously via autocrine or non-cell autonomously via targetderived paracrine, depending on the developmental stages of neurons [20-22]. While BDNF supplementation of culture media promotes the maturation of DNs, it may also mask defects in the endogenous BDNF action. In the present study, when BDNF was excluded from the culture media, ADHD-DN neurite development was significantly reduced when compared with that of Ctrl-DNs. These results indicate that the impaired neurite development exhibited by ADHD-DNs may involve defects in the endogenous BDNF action. 


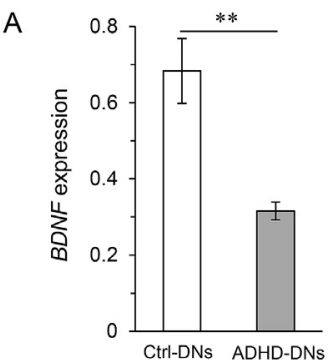

B

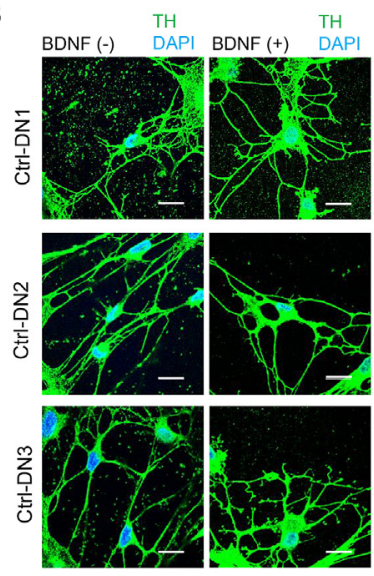

C

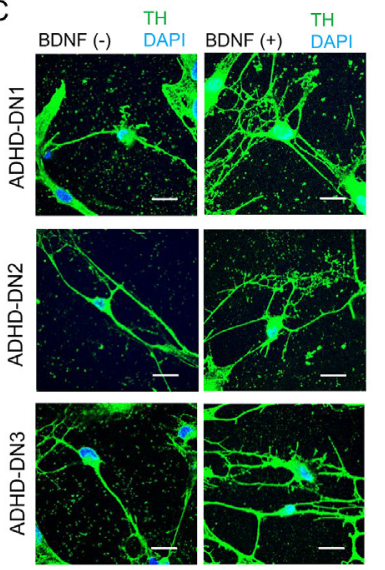

D

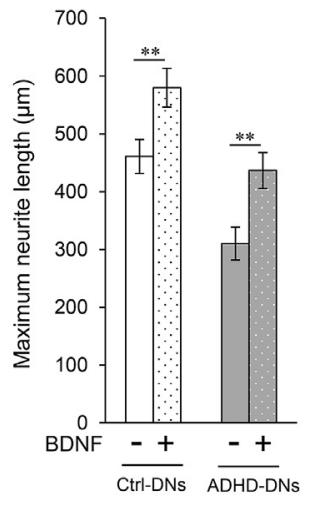

E

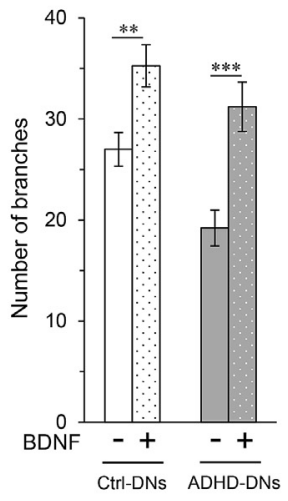

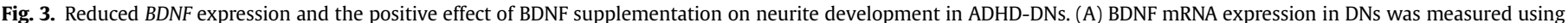

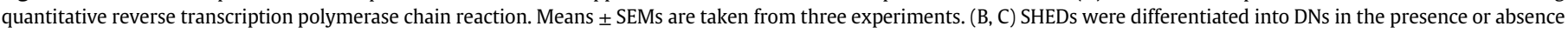

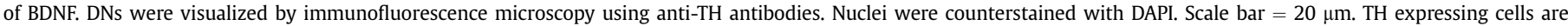

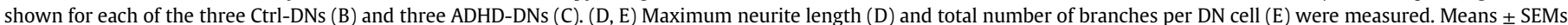
for 30 cells from each of the three Ctrl-DNs and ADHD-DNs are shown. ${ }^{* *} P<0.01 .{ }^{* * *} P<0.001$.

To test this, mRNA levels of BDNF were directly examined in ADHD-DNs and Ctrl-DNs. BDNF expression was significantly reduced in ADHD-DNs, suggesting transcriptional dysregulation of BDNF. BDNF secretion is regulated via many processes, including transcription, translation, post-translational processing, vesicle transporting, and exocytosis [12]. Furthermore, BDNF secretion is regulated both constitutively and in an activity-dependent manner in neurons [12]. A single nucleotide polymorphism at nucleotide 196 (G/A) located in the 5' pro-BDNF sequence, producing Val66Met, affects intracellular transport and activity-dependent secretion of BDNF and has been associated with psychopathology, including ADHD [13,14, and 23]. In the subjects used in the present study, no specific association was found between this polymorphism and ADHD phenotypes. Although the BDNF secretion is regulated by many factors, transcriptional dysregulation of BDNF may drive defects in endogenous BDNF action required for neurite development in ADHD-DNs.

Supplementation of the culture media with BDNF enhanced neurite development and mitochondrial function in ADHD-DNs in the present study. The positive effects of BDNF on neurons, including neurite development and mitochondrial function, result from signaling pathways that act via the tropomyosin receptor kinase B (TrkB) receptor [24]. cAMP responsive element binding protein (CREB), the transcription factor downstream of the TrkB receptor, induces the expression of genes encoding proteins, including peroxisome proliferator-activated receptor $\gamma$ coactivator$1 \alpha$ (PGC- $1 \alpha)$ and mitochondrial transcription factor A (TFAM), which activate mitochondrial biogenesis, motility, and the electron transport chain [25-28]. These proteins contribute to a positive feedback loop that further promotes the expression of BDNF [29]. This positive BDNF feedback loop may then be attenuated by reduced BDNF production in ADHD-DNs. However, the positive effect of exogenous BDNF on ADHD-DNs suggests no critical defect in the BDNF-TrkB signaling pathway in these cells. This further suggests that ADHD symptoms may be improved by exogenous BDNF.

The present study poses several limitations to a comprehensive understanding of the clinical manifestations of ADHD based on the pathological association between DNs, mitochondrial dysfunction, and BDNF changes. First, further studies are required to translate in vitro findings into clinical symptoms, such as the evaluation of intracerebral concentrations of dopamine and BDNF in subjects. Second, the mechanism(s) underlying the reduced $B D N F$ transcripts in ADHD-DNs has not yet been elucidated. Future work should assess the expression and activity of the transcription factors regulating BDNF transcription constitutively as well as in an activity-dependent manner (e.g., through neurotransmitters such as glutamate). Third, defects of other neuronal subsets, glial cells supporting neurons, and additional neurotrophic factors likely to be associated with ADHD neuropathology remain unclear. Further work should establish an experimental system for the induction of SHED differentiation into these cells for extensive analysis.

In conclusion, ADHD-DNs exhibited impaired neurite development and mitochondrial dysfunction in the present study. These phenotypes were further associated with decreased BDNF transcripts and were improved by exogenous BDNF administration. These results suggest the possibility that BDNF is a candidate therapeutic target for the treatment of intracerebral dopaminergic signaling deficits in children with ADHD. Thus, our findings in patient-derived SHED may contribute to the development of optimal personalized treatments for the early stages of ADHD. 
A

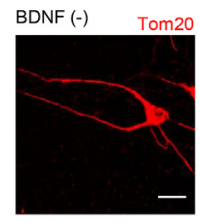

$\operatorname{BDNF}(+)$
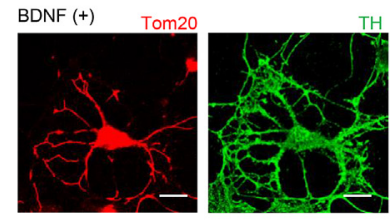

C
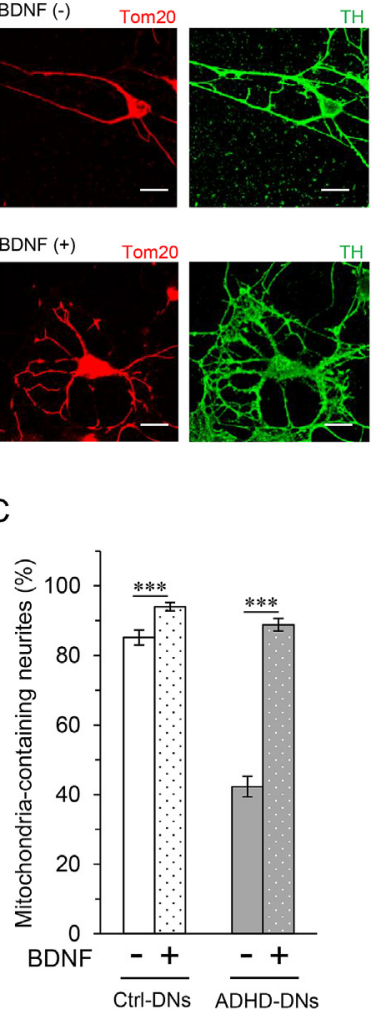

Ctrl-DN1
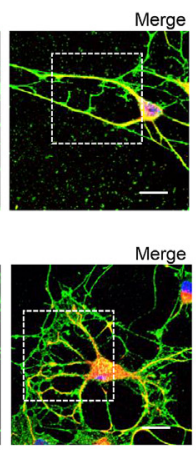

$\mathrm{D}$

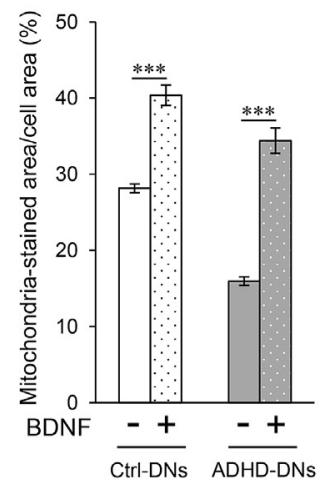

B
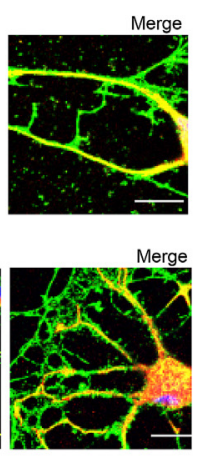

Merge

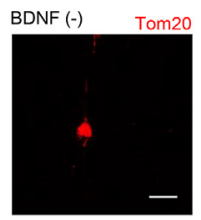

$\operatorname{BDNF}(+)$

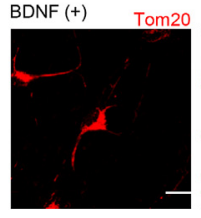

ADHD-DN1
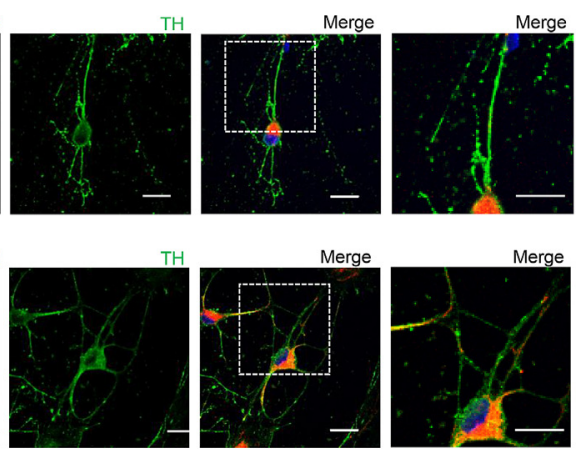

E

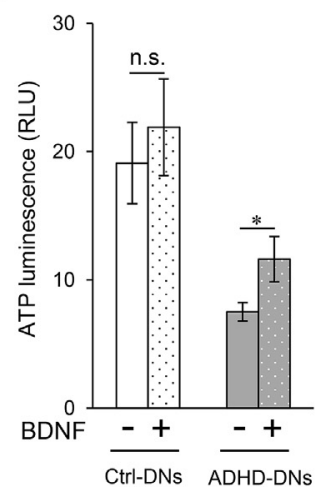

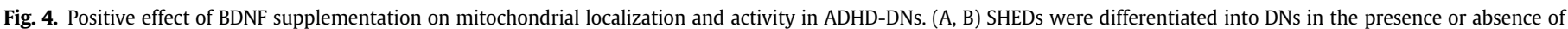

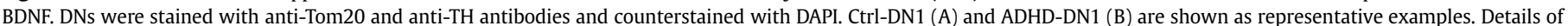

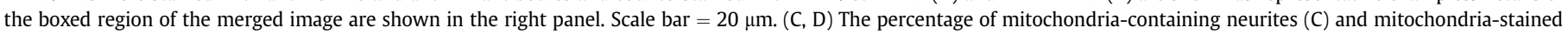

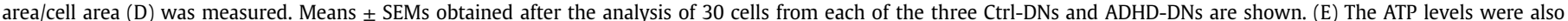

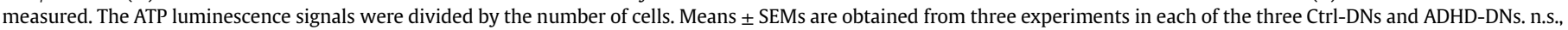
not significant. ${ }^{*} P<0.05$. ${ }^{* * *} P<0.001$.

\section{Conflicts of interest}

The authors have no conflicts of interest to declare.

\section{Acknowledgments}

We wish to thank all members of the Department of Pediatric and Special Needs Dentistry at Kyushu University Hospital for their valuable suggestions, technical support, and sharing of materials. We appreciate the technical assistance provided by the Research Support Center at the Research Center for Human Disease Modeling, Kyushu University Graduate School of Medical Sciences. This work was supported by the Japan Society for the Promotion of Science (KAKENHI grant numbers JP25670877, JP16K15839).

\section{Appendix A. Supplementary data}

Supplementary data to this article can be found online at https://doi.org/10.1016/j.bbrc.2019.04.084.

\section{References}

[1] A. Thapar, M. Cooper, Attention deficit hyperactivity disorder, Lancet 387 (2016) 1240-1250.

[2] E. Taylor, Developmental neuropsychopathology of attention deficit and impulsiveness, Dev. Psychopathol. 11 (1999) 607-628.

[3] S. Schmidt, F. Petermann, Developmental psychopathology: attention deficit hyperactivity disorder (ADHD), BMC Psychiatry 9 (2009) 58.

[4] S.V. Faraone, R.H. Perlis, A.E. Doyle, J.W. Smoller, J.J. Goralnick, M.A. Holmgren,
P. Sklar P, Molecular genetics of attention-deficit/hyperactivity disorder, Biol. Psychiatry 57 (2005) 1313-1323.

[5] J. Tarver, D. Daley, K. Sayal, Attention-deficit hyperactivity disorder (ADHD): an updated review of the essential facts, Child Care Health Dev. 40 (2014) $762-774$.

[6] F.X. Castellanos, R. Tannock, Neuroscience of attention-deficit/hyperactivity disorder: the search for endophenotypes, Nat. Rev. Neurosci. 3 (2002) $617-628$.

[7] M.O. Klein, D.S. Battagello, A.R. Cardoso, D.N. Hauser, J.C. Bittencourt, R.G. Correa, Dopamine: functions, signaling, and association with neurological diseases, Cell. Mol. Neurobiol. 1 (2019) 31-59.

[8] D. Viggiano, D. Vallone, A. Sadile, Dysfunctions in dopamine systems and ADHD: evidence from animals and modeling, Neural Plast. 11 (2004) 97-114.

[9] S.V. Faraone, The pharmacology of amphetamine and methylphenidate: relevance to the neurobiology of attention-deficit/hyperactivity disorder and other psychiatric comorbidities, Neurosci. Biobehav. Rev. 87 (2018) 255-270.

[10] P. Verma, A. Singh, D.N. Nthenge-Ngumbau, U. Rajamma, S. Sinha, K. Mukhopadhyay, K.P. Mohanakumar, Attention deficit-hyperactivity disorder suffers from mitochondrial dysfunction, BBA Clin 6 (2016) 153-158.

[11] A.M. Davies, E.M. Wright, Neurotrophic factors. Neurotrophin autocrine loops, Curr. Biol. 5 (1995) 723-726.

[12] V. Lessmann, T. Brigadski, Mechanisms, locations, and kinetics of synaptic BDNF secretion: an update, Neurosci. Res. 65 (2009) 11-22.

[13] D.Y. Liu, X.M. Shen, F.F. Yuan, O.Y. Guo, Y. Zhong, J.G. Chen, L.Q. Zhu, J. Wu, The physiology of BDNF and its relationship with ADHD, Mol. Neurobiol. 52 (2015) $1467-1476$.

[14] S.J. Tsai, Role of neurotrophic factors in attention deficit hyperactivity disorder, Cytokine Growth Factor Rev 34 (2017) 35-41.

[15] J. Wang, X. Wang, Z. Sun, X. Wang, H. Yang, S. Shi, S. Wang, Stem cells from human-exfoliated deciduous teeth can differentiate into dopaminergic neuron-like cells, Stem Cell. Dev. 19 (2010) 1375-1383.

[16] H. Fujii, K. Matsubara, K. Sakai, M. Ito, K. Ohno, M. Ueda, A. Yamamoto, Dopaminergic differentiation of stem cells from human deciduous teeth and their therapeutic benefits for Parkinsonian rats, Brain Res. 1613 (2015) 59-72.

[17] S. Hirofuji, Y. Hirofuji, H. Kato, K. Masuda, H. Yamaza, H. Sato, F. Takayama, M. Torio, Y. Sakai, S. Ohga, T. Taguchi, K. Nonaka, Mitochondrial dysfunction in 
dopaminergic neurons differentiated from exfoliated deciduous tooth-derived pulp stem cells of a child with Rett syndrome, Biochem. Biophys. Res. Commun. 498 (2018) 898-904.

[18] T.T.M. Pham, H. Kato, H. Yamaza, K. Masuda, Y. Hirofuji, H. Sato, H.T.N. Nguyen, X. Han, Y. Zhang, T. Taguchi, K. Nonaka, Altered development of dopaminergic neurons differentiated from stem cells from human exfoliated deciduous teeth of a patient with Down syndrome, BMC Neurol. 18 (2018) 132.

[19] H.T.N. Nguyen, H. Kato, K. Masuda, H. Yamaza, Y. Hirofuji, H. Sato, T.T.M. Pham, F. Takayama, Y. Sakai, S. Ohga, T. Taguchi, K. Nonaka, Impaired neurite development associated with mitochondrial dysfunction in dopaminergic neurons differentiated from exfoliated deciduous tooth-derived pulp stem cells of children with autism spectrum disorder, Biochem. Biophys. Rep. 16 (2018) 24-31.

[20] E.M. Wright, K.S. Vogel, A.M. Davies, Neurotrophic factors promote the maturation of developing sensory neurons before they become dependent on these factors for survival, Neuron 9 (1992) 139-150.

[21] P.L. Cheng, A.H. Song, Y.H. Wong, S. Wang, X. Zhang, M.M. Poo, Self-amplifying autocrine actions of BDNF in axon development, Proc. Natl. Acad. Sci. U.S.A 108 (2011) 18430-18435.

[22] C. Sampathkumar, Y.J. Wu, M. Vadhvani, T. Trimbuch, B. Eickholt, C. Rosenmund, Loss of MeCP2 disrupts cell autonomous and autocrine BDNF signaling in mouse glutamatergic neurons, Elife 5 (2016), e19374.
[23] M.F. Egan, M. Kojima, J.H. Callicott, T.E. Goldberg, B.S. Kolachana, A. Bertolino E. Zaitsev, B. Gold, D. Goldman, M. Dean, B. Lu, D.R. Weinberger, The BDNF val66met polymorphism affects activity-dependent secretion of BDNF and human memory and hippocampal function, Cell 112 (2003) 257-269.

[24] E.J. Huang, L.F. Reichardt, Trk receptors: roles in neuronal signal transduction, Annu. Rev. Biochem. 72 (2003) 609-642.

[25] T. Pizzorusso, G.M. Ratto, E. Putignano, L. Maffei, Brain-derived neurotrophic factor causes cAMP response element-binding protein phosphorylation in absence of calcium increases in slices and cultured neurons from rat visual cortex, J. Neurosci. 20 (2000) 2809-2816.

[26] S. Herzig, F. Long, U.S. Jhala, S. Hedrick, R. Quinn, A. Bauer, D. Rudolph, G. Schutz, C. Yoon, P. Puigserver, B. Spiegelman, M. Montminy, CREB regulates hepatic gluconeogenesis through the coactivator PGC-1, Nature 413 (2001) $179-183$.

[27] S.M. Raefsky, M.P. Mattson, Adaptive responses of neuronal mitochondria to bioenergetic challenges: roles in neuroplasticity and disease resistance, Free Radic. Biol. Med. 102 (2017) 203-216.

[28] Z. Xu, X.A. Lv, Q. Dai, M. Lu, Z. Jin, Exogenous BDNF increases mitochondrial pCREB and alleviates neuronal metabolic defects following mechanical injury in a MPTP-dependent way, Mol. Neurobiol. 55 (2018) 3499-3512.

[29] J. Tuvikene, P. Pruunsild, E. Orav, E.E. Esvald, T. Timmusk, AP-1 transcription factors mediate BDNF-positive feedback loop in cortical neurons, J. Neurosci. 36 (2016) 1290-1305. 


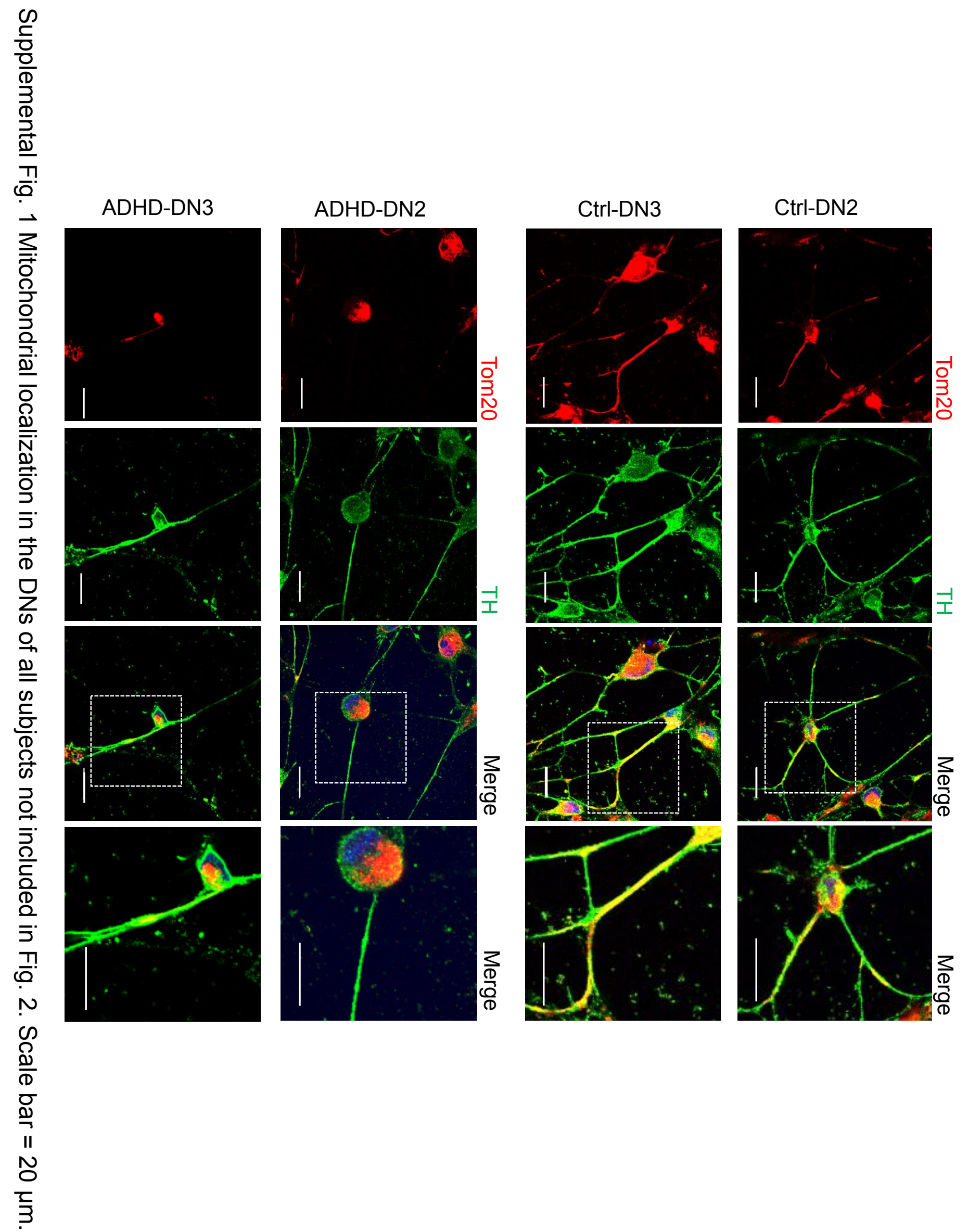




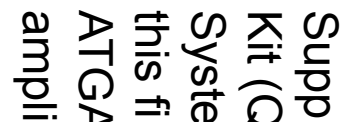

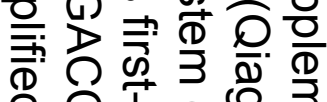

음

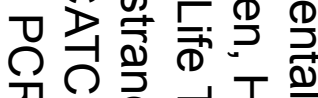

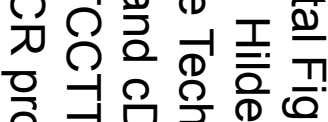

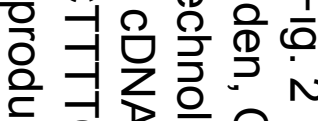

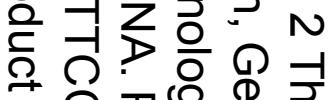

$\sum \bigcirc 0$ 을.

क्.

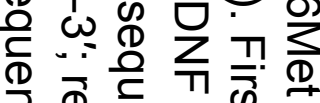

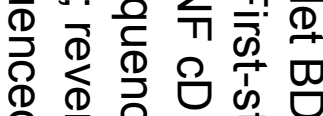

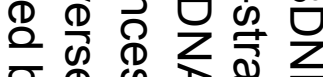

व

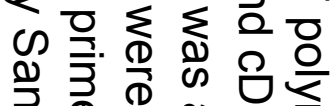

은

两 웅

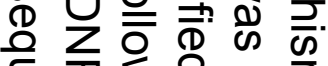

更

을. 웅

织 $\bigcirc$

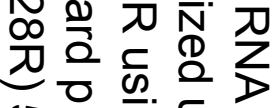

을. 등

○े

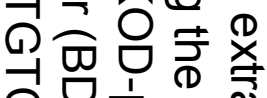

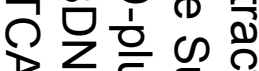

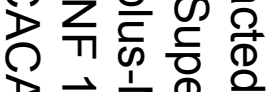

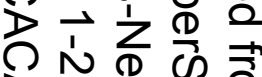

긍유으을 을

Q v후용

ᄀํ.
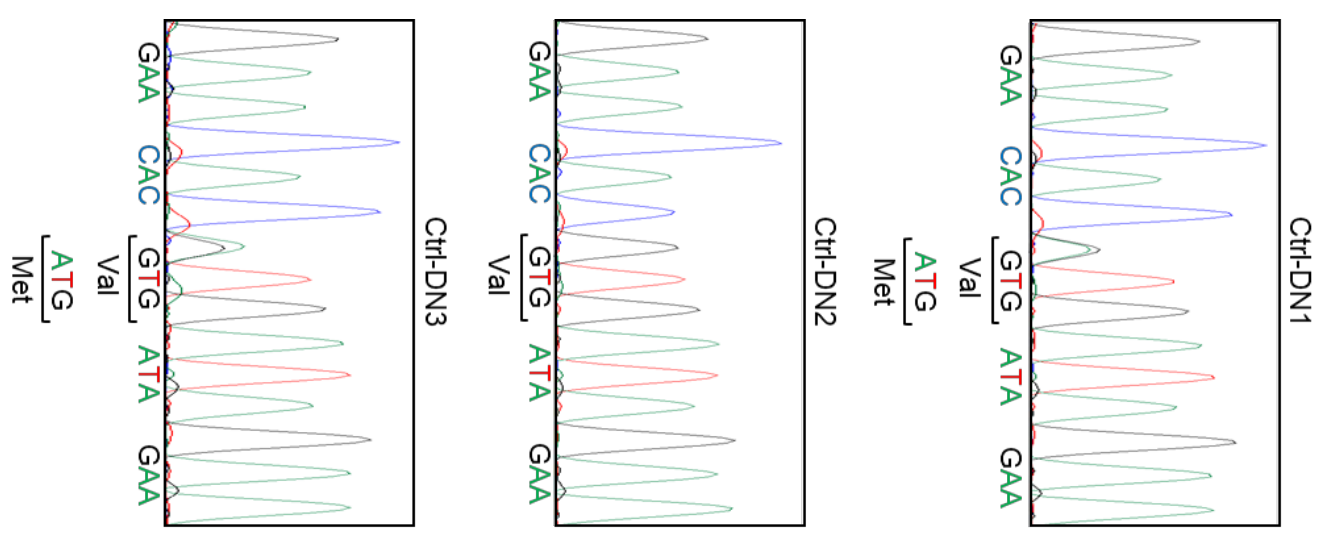

ก 8 ㄲ

म $\overline{\frac{1}{0}}$

ค क जि

ค )

山.

굼 夋 心

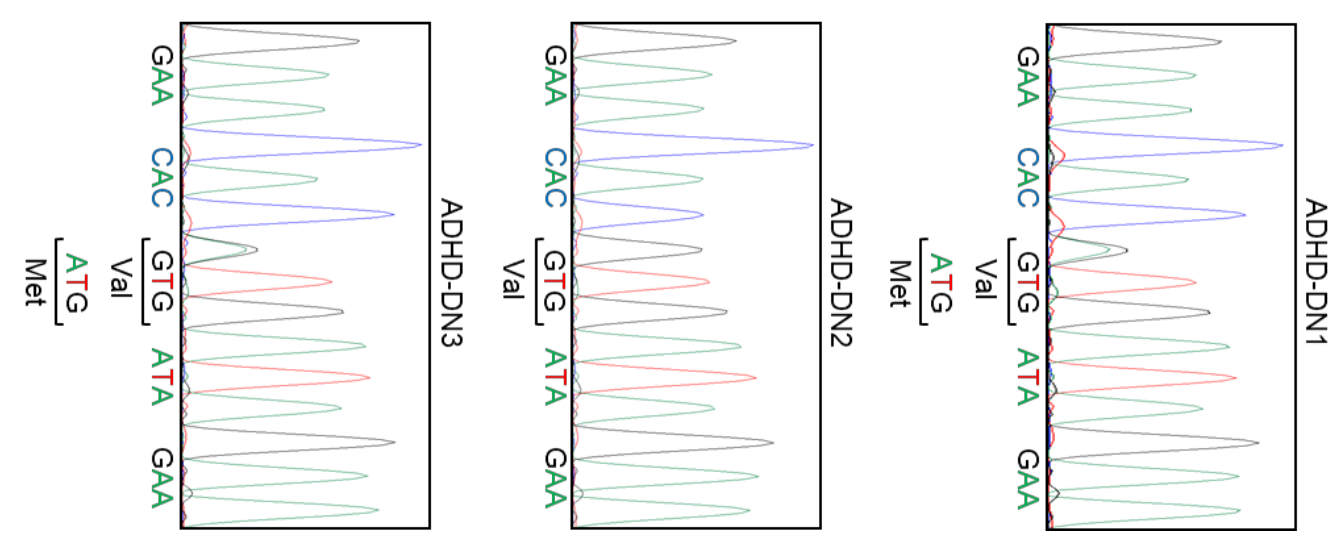

(1) 党豆

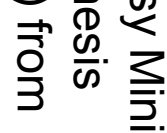




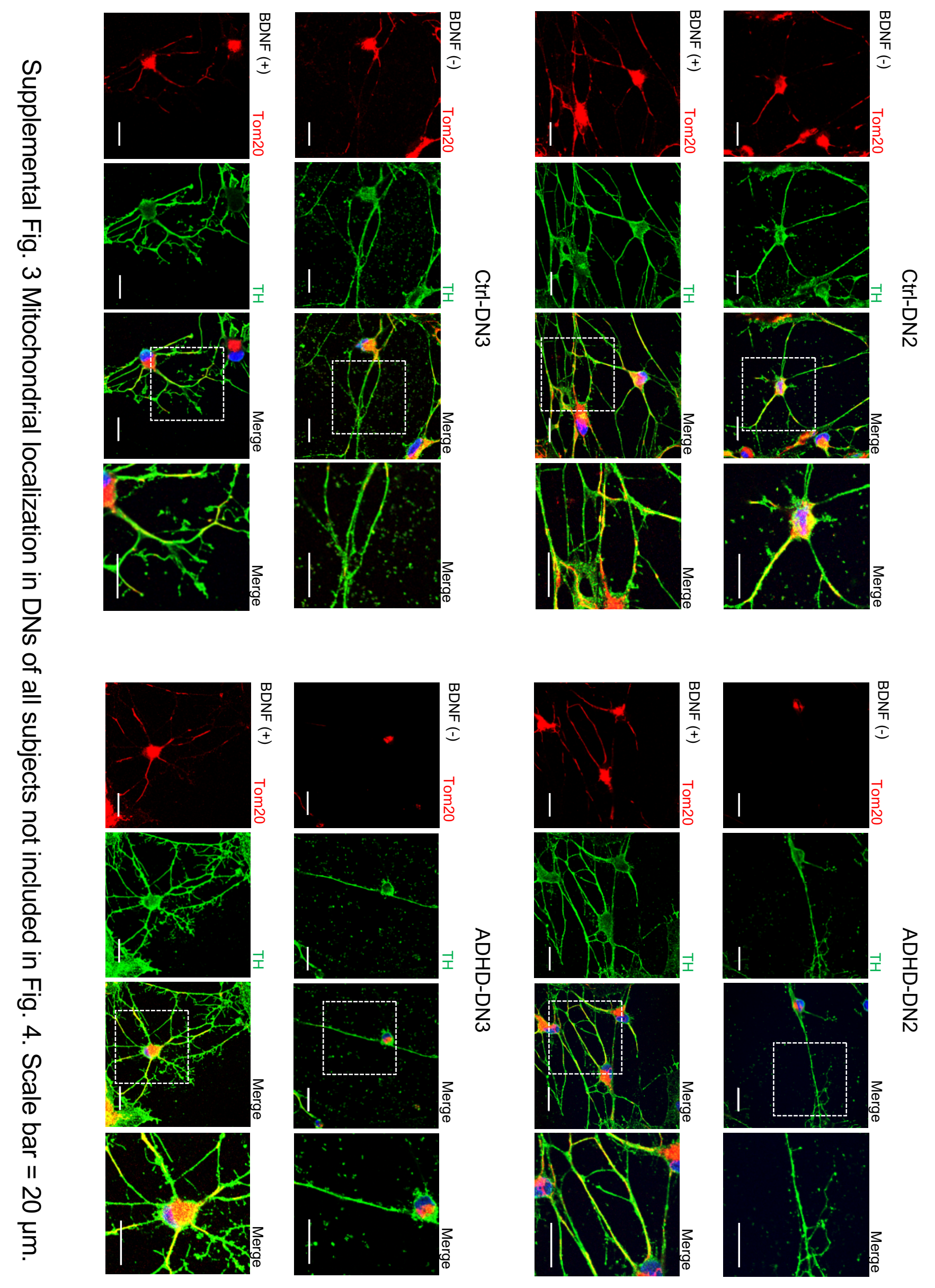

